\title{
TRADUCCIÓN, VALIDACIÓN Y ADAPTACIÓN TRANSCULTURAL DEL SCHOOLAGERS' COPING STRATEGIES INVENTORY PARA ESCOLARES COLOMBIANOS
}

\author{
Cross-cultural translation, validation and adaptation of the Schoolagers' Coping \\ Strategies Inventory for Colombian schoolchildren
}

\section{Tradução, validação e adaptação transcultural do Schoolagers' Coping Strategies Inventory para escolares colombianos}

\author{
Karol Johanna Briñez Ariza (OrcID) \\ Pontificia Universidad Javeriana - PUJ - Bogotá - Colombia
}

Clara Virginia Caro Castillo (OrcID)

Universidad Nacional de Colombia - UN - Bogotá - Colombia

Marta Lenise Do Prado (OrcID)

Universidad Federal de Santa Catarina - UFSC - Florianópolis (SC) - Brasil

\section{RESUMEN}

Objetivo: Realizar la traducción, validación y adaptación transcultural del Schoolagers' Coping Strategies Inventory (Inventario de Estrategias de Afrontamiento de Escolares) (SCSI) con estudiantes colombianos. Métodos: Estudio metodológico realizado entre 2015 y 2016 con alumnos entre 8 y 15 años de edad, de instituciones de enseñanza públicas y privadas de Ibagué, Colombia. Se utilizó la ficha para caracterización de la muestra y el SCSI. La validación transcultural ha sido organizada en ocho etapas: la traducción oficial para el español; la traducción del original para el inglés; la traducción reversa; la versión consensual; la validez facial y la adaptación al idioma de parte de los estudiantes; la validez facial y de contenido por especialistas; la versión final; la prueba piloto y de confiabilidad. Resultados: La versión original en inglés ha sido adaptada para el idioma español de Colombia con la intervención de estudiantes, de extractos socioeconómicos altos y bajos con grupos focales y prueba piloto, utilizando sus expresiones verbales y cambiando 4 ítems; y con la colaboración de especialistas en investigación metodológica y en coping, también cambiando 4 ítems, con confiabilidad de 0,80. Conclusión: EI SCSI ha sido traducido, validado y adaptado transculturalmente para la población colombiana. La versión obtenida fue el SCSI-VCONF lo cual se mostró comprensible para la población investigada, válida y confiable para investigaciones con escolares para determinar la frecuencia de uso y la efectividad de estrategias de afrontamiento.

Descriptores: Estudios de Validación; Encuestas y Cuestionarios; Investigación Metodológica en Enfermería; Adaptación Psicológica.

\section{ABSTRACT}

Objective: To carry out the cross-cultural validation and adaptation of the Schoolagers' Coping Strategies Inventory (SCSI) with Colombian students. Methods: Methodology research carried out from 2015 to 2016 with students aged 8 to 15 years from public and private schools in Ibagué, Colombia. A characterization form and the SCSI were used. The cross-cultural validation was organized in eight stages: official translation into Spanish, translation of the original into English, reverse translation, consensus version, facial validity and language adaptation by students, facial and content validity by experts, final version, pilot study and reliability. Results: the original English version was adapted to the Spanish and Colombian language with the help from students of high and low socioeconomic statuses in focus groups and pilot study using their verbal expressions and modifying 4 items and with the collaboration of experts in methodological research and coping who also modified 4 items. Reliability was 0.80. Conclusion: SCSI was cross-culturally validated and adapted for the Colombian population. The final version was named SCSIVCONF and was understandable to the target population, valid and reliable for research with schoolchildren to determine the frequency of use and effectiveness of coping strategies.

Descriptors: Validation Studies; Surveys and Questionnaires; Nursing Methodology Research; Adaptation, Psychological. 


\section{RESUMO}

Objetivo: Realizar a tradução, validação e adaptação transcultural do Schoolagers' Coping Strategies Inventory (Inventário de Estratégias de Enfrentamento de Escolares) (SCSI) com estudantes colombianos. Métodos: Estudo metodológico realizado com alunos de 8 a 15 anos de idade, de instituições de ensino públicas e privadas, em Ibagué, Colômbia. Utilizou-se a ficha de caracterização e o SCSI. A validação transcultural foi organizada em oito etapas: tradução oficial para o espanhol; tradução do original para o inglês; tradução reversa; versão consensual; validade facial e adaptação à língua pelos estudantes; validade facial e de conteúdo por especialistas; versão final; teste piloto e confiabilidade. Resultados: $A$ versão original em inglês foi adaptada para o idioma espanhol colombiano, com a intervenção de estudantes, de estratos socioeconômicos altos e baixos, em grupos focais e teste piloto, utilizando suas expressões verbais e modificando 4 itens; e com a colaboração de especialistas em pesquisa metodológica e em coping, também modificando 4 itens, com confiabilidade de 0,80. Conclusão: O SCSI foi traduzido, validado e adaptado transculturalmente para a população colombiana. A versão obtida foi o SCSI-VCONF, o qual mostrou-se compreensível para a população-alvo e válida e confiável para pesquisas com escolares para determinar a frequência de uso e efetividade de estratégias de enfrentamento.

Descritores: Estudos de Validação; Inquéritos e Questionários; Pesquisa Metodológica em Enfermagem; Adaptação Psicológica.

\section{INTRODUCCIÓN}

Las intervenciones de cuidado de enfermería de niños y adolescentes deben originarse en la experiencia y necesidades específicas en los momentos estresantes y sus respuestas humanas, porque son puente para desarrollar estrategias que puedan reducir el estrés y mejorar el afrontamiento ${ }^{(1)}$.

El afrontamiento son pensamientos reales, flexibles y actos que resuelven, gestionan problemas y reducen estrés ${ }^{(2,3)}$ conceptualizado a partir de dos atributos: esfuerzos (lo que se hace) y función (para qué). Los esfuerzos provienen tanto de procesos cognitivos/psicológicos, como conductuales/comportamentales que envuelven emociones y acciones ${ }^{(2)}$. Las funciones del afrontamiento son reconocidas para gestionar demandas externas e internas, porque desbordan los recursos individuales ante el estrés ${ }^{(4,5)}$; para mitigar los efectos adversos del estrés y como preparador para la acción ${ }^{(2)}$; para adaptarse y hacer transición en procesos de enfermedad crónica ${ }^{(5)}$; y para comprender las respuestas a las situaciones de cambio en la enfermedad y la salud(6).

En enfermería se cuenta con el modelo conceptual de adaptación que incluye el concepto de procesos de afrontamiento, comprendido como formas innatas o adquiridas de contestar al ambiente cambiante del sistema adaptativo humano ${ }^{(7)}$ y desde la teoría de mediano rango de afrontamiento( ${ }^{(8)}$ y las escalas de medición ${ }^{(6)}$. Los procesos innatos son genéticamente determinados o comunes a las especies, y son vistos generalmente como procesos automáticos. Los procesos de afrontamiento adquiridos son desarrollados a través de estrategias por aprendizaje. Las experiencias encontradas durante la vida contribuyen a las habituales respuestas a un estímulo particular ${ }^{(7)}$.

Las estrategias de afrontamiento son el uso de pensamientos y comportamientos para enfrentar preocupación o estrés. Pueden modificarse con el tiempo y ser inherentes o aprendidas ${ }^{(9)}$. En razón a que las características individuales como edad y género, experiencias y el ambiente influencian en los niños la selección de sus estrategias de afrontamiento. Es asumido que el afrontamiento en general tiene características predecibles entre los grupos de niños ${ }^{(4,9)}$.

La medición de estrategias de afrontamiento es necesaria en niños. Los instrumentos de medición del afrontamiento ayudan a identificar las estrategias usadas para lidiar con el estrés ${ }^{(10)}$ y su categorización depende de la medida usada $^{(2)}$. La mayor investigación se ha enfocado en adultos y adolescentes así como los instrumentos propuestos para ello.

El afrontamiento es una ficha clave en la promoción de la salud ${ }^{(8)}$. Comprendiendo el uso de las estrategias del afrontamiento y su efectividad en niños pueden conocerse sus comportamientos ante situaciones de preocupación para ellos.

Se encontraron varios instrumentos disponibles en la literatura, aunque ninguno para ser usado en escolares desde los ocho años y publicados en otros idiomas distintos al español. Se encontró el Schoolagers' Coping Strategies Inventory (SCSI) con versión en inglés ${ }^{(9)}$, lo que hizo necesario desarrollar etapas de traducción y validación, que permitieran medir un fenómeno similar en culturas que no son las mismas y lograr hacer comparaciones ${ }^{(10-12)}$. 
Este artículo tiene relevancia para el conocimiento porque mediante metodología científica se hizo adaptación transcultural de un indicador empírico útil que permitirá medir un concepto de la teoría de enfermería de afrontamiento, pertinente para la investigación en diferentes niveles de evidencia de los fenómenos de cuidado en la edad escolar.

EI SCSI evalúa el uso y la efectividad de estrategias de afrontamiento en escolares y se presenta válido y confiable ${ }^{(9,13-15)}$. El objetivo del estudio fue realizar la traducción, validación y adaptación transcultural del SCSI para la población de escolares colombianos.

\section{MÉTODOS}

Estudio metodológico realizado entre 2015 y 2016 con escolares de 8 a 15 años de edad, de instituciones educativas públicas y privadas de Bogotá e Ibagué, Colombia. Para la recolección de la información se usó la ficha de caracterización de escolares y SCSI. Fueron seleccionados de dos ciudades en dos niveles socioeconómicos diferentes para comparar si usaban el mismo lenguaje.

La ficha de caracterización de los escolares, propuesta por la autora principal, contenía opciones de respuesta para variables sexo, edad, grado, tipo de institución educativa. Fue aplicada en dos grupos distintos: para validez de facial en grupos focales y en los escolares que participaron en la prueba piloto.

SCSI: Instrumento de medición para niños en edad escolar que mide estrategias de afrontamiento (frecuencia de uso y efectividad) durante situaciones estresantes ${ }^{(12)}$. Compuesto por 34 ítems, organizado en dos subescalas: la subescala de uso y la de efectividad. La subescala de uso, mide cuánto (frecuencia) el escolar usa esa estrategia de afrontamiento cuando está preocupado o estresado. Cada ítem es evaluado en escala tipo likert con opciones de respuesta: nunca (0), de vez en cuando (1), muchas veces (2), y siempre (3). La subescala de efectividad, mide cuanto ayuda al escolar usar esa estrategia de afrontamiento cuando esta estresado o preocupado. Cada ítem es evaluado en escala tipo likert con opciones de respuesta: no me ayuda (0), me ayuda poco (1), y me ayuda mucho (2). El puntaje total de la subescala de uso de las estrategias de afrontamiento oscila entre 0 a 102 y de la subescala de efectividad entre 0 a 68. La puntación global de la escala varía de 0 a 170 . Cuanto mayor puntaje mayor frecuencia del uso de estrategias de afrontamiento y mayor su efectividad ante situaciones de estrés o preocupación ${ }^{(14)}$.

El proceso de adaptación transcultural fue organizado en ocho etapas ${ }^{(11,12,16,17)}$, presentadas en la Figura 1. Cada una de las etapas será descrita en la sección de los resultados.

Figura 1 - Proceso de validación transcultural SCSI para español de Colombia.

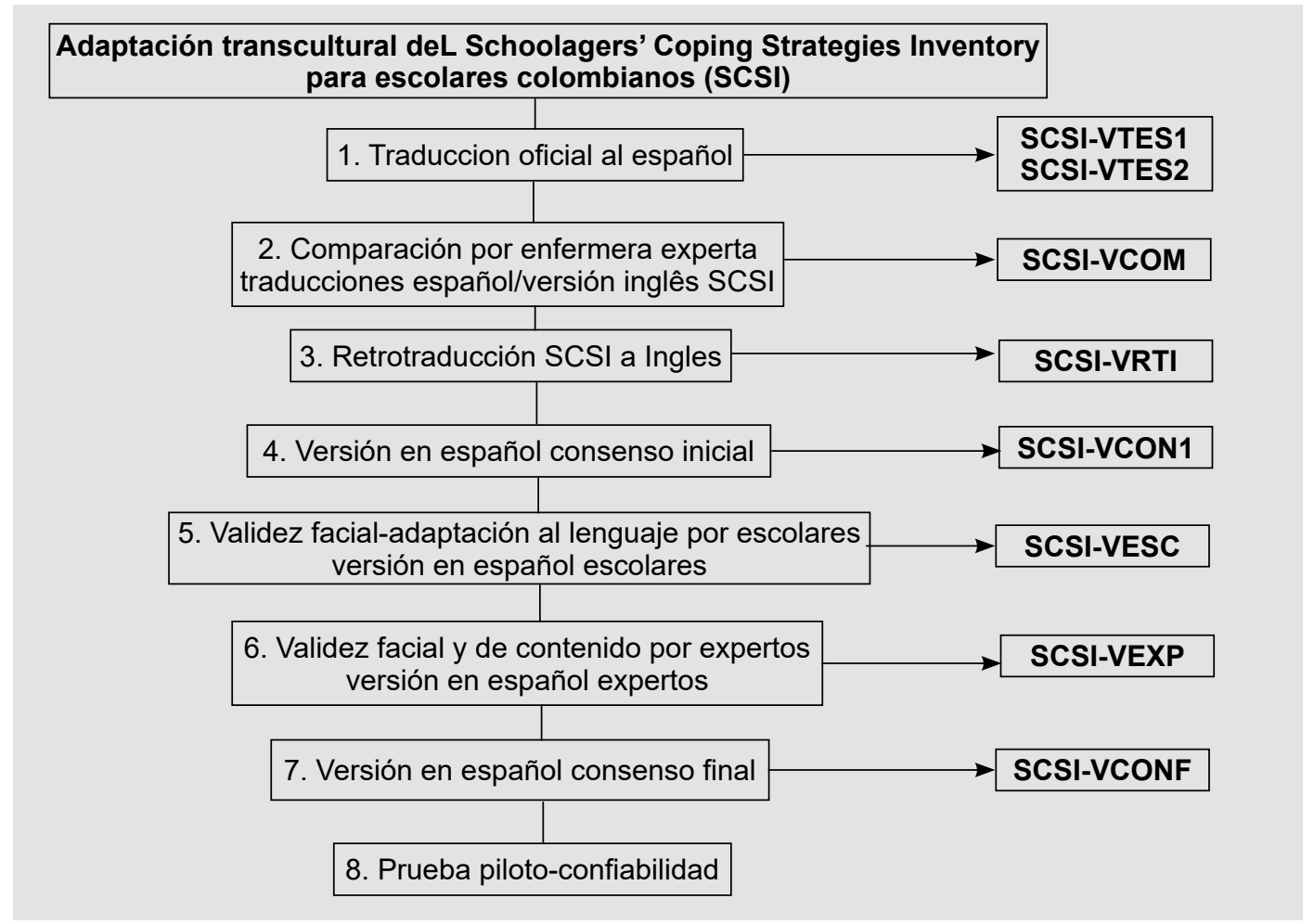

Fuente: elaboración propia. 
De acuerdo con el Ministerio de Salud(18) en la Resolución 8430 de 1993, esta investigación se consideró con riesgo mínimo. La investigación fue aprobada por el Comité de Ética de la Facultad de enfermería de la Universidad Nacional de Colombia, las Secretarías de Educación y de Salud de Ibagué y una institución educativa en Bogotá.

En la fase de validez facial, y adaptación al lenguaje se solicitaron consentimientos informados a los padres de los escolares mediante y asentimiento informados a los escolares, así como se solicitó autorización de participación a los expertos:

1. Traducción oficial al español: Realizada después de recibir la autorización por parte de la autora de la versión original en inglés ${ }^{(9)}$. El objetivo de esta etapa fue obtener dos traducciones oficiales para el español de Colombia. Fueron realizadas de forma independiente por dos traductores oficiales de la Universidad Nacional de Colombia, uno con idioma nativo inglés y el otro, con idioma nativo español.

2. Comparación de las traducciones: Comparación por enfermera experta de las traducciones oficiales en español del SCSI con la versión original en inglés. El objetivo de esta etapa fue comparar las traducciones en español del SCSI con la versión original en inglés. Para esto, se presentaron las dos traducciones oficiales junto con la versión original en inglés a una enfermera con dominio del inglés y del español, experta en la metodología de validación de instrumentos de medición. Fue solicitado que, a partir de las versiones, fuese elaborada una versión consensual que presentara el lenguaje cotidiano colombiano y fuera comprensible para escolares. Las dos traducciones al español se compararon con la versión original en inglés generando una versión para escolares SCSI-VCOM.

3. Retrotraducción: El objetivo de esta etapa fue traducir la versión producida en la segunda etapa SCSI-VCOM para el idioma original del instrumento. Fue contactado un tercer traductor oficial de la Universidad Nacional de Colombia con idioma nativo inglés, a quien le fue solicitada la respectiva retrotraducción del español al inglés, resultando la versión SCSI-VRTI.

4. Versión de consenso inicial en el español colombiano: El objetivo de esta etapa fue definir una versión para escolares que incluyera las recomendaciones identificadas en las tres etapas anteriores (SCSI-VTES (1+2) + SCSI-VCOM + SCSI-VTRI). Se organizó una reunión para la elaboración de la versión consensual, que tuvo como participantes a los investigadores. La etapa concluyó en la elaboración de la versión SCSI-VCON1.

5. Validez facial y adaptación al lenguaje de los escolares: El objetivo de esta etapa fue que la versión consensual SCSI-VCON1 contemplara las expresiones verbales de los escolares en los ítems del instrumento. Se realizó mediante una estrategia cualitativa de grupos focales acorde a la población destino presentando imágenes por medio de un proyector de diapositivas mostrando cada ítem del SCSI-VCON1 y preguntando "como conversaban y manifestaban esas frases" en el lenguaje cotidiano colombiano los escolares de ocho a diez años. Cada discusión fue grabada en audio y después transcrita para el análisis. Se diseñó un documento sustentando los grupos focales presentado a los rectores de las Instituciones Educativas con el fin de apoyar teórica y metodológicamente el desarrollo de esta estrategia ${ }^{(19,20,21)}$. Fueron aplicadas preguntas de apertura, introductorias, de transición, clave, y se finalizó con una merienda para los participantes y retroalimentación.

6. Validez facial y de contenido por expertos: El objetivo de esta etapa fue obtener la evaluación del SCSI-VESC por seis expertos de diferentes Universidades de Colombia con trayectoria en la adaptación de instrumentos de medición, experiencia profesional y de investigación en pediatría y el tema de afrontamiento. Se obtuvo autorización de la planilla "juicio de expertos"(22) diligenciada por cada experto para evaluar cinco categorías en validez de contenido: equivalencia semántica, claridad, coherencia, relevancia y suficiencia con puntaje de 1 (no cumple) a 4 (alto nivel de calificación). En validez de apariencia, evaluaron la redacción de ítems, la exactitud y la claridad del lenguaje con puntaje de 0 (no cumple) y 1 (cumple). Para la interpretación de los valores del kappa se siguió el referente teórico, el cual refiere la fuerza de concordancia así: pobre $(0,00)$; leve $(0,01-0,20)$; aceptable $(0,21-0,40)$; moderada $(0,41-0,60)$; considerable $(0,61-0,80)$; casi perfecta $(0,81-1,00)$. Para el análisis de la validez de apariencia se calculó porcentaje de acuerdo entre expertos en cumplimiento de criterios de redacción, exactitud y claridad en el lenguaje.

7. Versión consenso final: El objetivo de esta etapa fue tomar decisiones para la adecuación semántica de los ítems, según las sugerencias recibidas en las etapas quinta y sexta. El consenso se realizó por un comité entre la investigadora principal, la directora de la tesis doctoral, dada su experiencia en el trabajo profesional con escolares y el estadístico. 
8. Prueba piloto: El objetivo de esta etapa fue verificar la comprensión de los ítems, e instrucciones en el diligenciamiento de los instrumentos, necesidad de apoyo y materiales, cálculo del tiempo para su aplicación y así realizar modificaciones y/o correcciones pertinentes. Se ubicó en puesto individual y separado a cada escolar y le fue entregado el formato del SCSI-VCONF y un bolígrafo negro por la investigadora principal y un auxiliar de investigación. Para la confiabilidad se calculó el alfa de Cronbach, siendo considerados

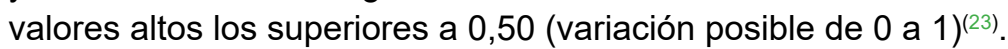

Si bien el objetivo fue una evaluación cualitativa en cuanto a la comprensión de los ítems por participantes de la misma población en la que el instrumento SCSI-VCONF iba a ser aplicada, se calculó el alfa de Cronbach, comprendiendo que era una muestra pequeña. Sin embargo, otras investigaciones futuras ligadas al presente estudio metodológico podrán sustentar las propiedades psicométricas del instrumento SCSI-VCONF.

\section{RESULTADOS}

Traducción oficial al español: Esta etapa produjo dos versiones: la SCSI-VTES1 y la SCSI-VTES2.

Comparación de las traducciones: Los ajustes se hicieron al identificar que tenían diferencias en la presentación de los ítems. Se observó que en una de las traducciones los ítems fueron traducidos de manera más personal facilitando que el escolar pudiera verse reflejado en esas estrategias de afrontamiento. Por ejemplo, algunas de las comparaciones en los ítems entre las versiones fueron: en el ítem 3, SCSI-VTES1 tradujo "morder mis uñas" y SCSI-VTES2 tradujo "me como las uñas"; en el ítem 17, SCSI-VTES1 tradujo "esperar lo mejor" y SCSI-VTES2 tradujo "espero que salga bien"; en el ítem 24, SCSI-VTES1 tradujo "resolver el problema" y SCSI-VTES2 tradujo "resuelvo el problema", el lenguaje fue comprensible pero requería validez facial por escolares. De esta forma fue considerada la versión más entendible para los escolares que fue la de la redacción más personalizada, produciendo la versión SCSI-VCOM.

Validez facial y adaptación al lenguaje de los escolares: Participaron 42 escolares con edades entre ocho y 10 años divididos en cinco grupos focales, de tercer grado de primaria.

Del total, 11 eran de sexo femenino y 14 de sexo masculino, pertenecientes a una institución estatal de Bogotá de nivel socio económico bajo, y 9 de sexo femenino y 8 de sexo masculino, de una institución educativa no estatal de lbagué de nivel socioeconómico alto.

Los 34 ítems de SCSI-VCON1 fueron presentados a los escolares y ellos los adecuaron al lenguaje cotidiano. Las propuestas de los integrantes del grupo focal recibían votación y sumado a discusión ellos mismos decidían cual frase era la más clara y apropiada para cada ítem. Se destaca que veinticuatro ítems no sufrieron cambios en su enunciado por la facilidad de su comprensión. Cuatro ítems fueron modificados por los escolares para ser redactados de manera similar, pero más comprensible al usar palabras de uso cotidiano en lenguaje colombiano, y tiempo verbal presente, o ajustes que indicaran que era algo propio (Ver Tabla I). Los cambios fueron organizados en la versión SCSI-VESC.

Tabla I - Ajustes sugeridos por los escolares en algunas palabras de cuatro enunciados de los ítems del SCSI. Colombia.

\begin{tabular}{cll}
\hline Ítem $\mathbf{N}^{\circ}$ & Ítems versión (SCSI- VCON1) & Ítems cambiados por los escolares (SCSI-VESC) \\
\hline $\mathbf{2}$ & Busco estar solo & Estoy solo \\
$\mathbf{3}$ & Me muerdo las uñas & Me como las uñas \\
$\mathbf{2 5}$ & Dejo de pensar en ello & Dejo de pensar en eso \\
$\mathbf{3 1}$ & Pienso en ello & Pienso en eso \\
\hline
\end{tabular}

Fuente: Briñez K, 2016

Validez facial y de contenido por expertos: Después de realizar la entrega de las versiones SCSI-VCON1 y SCSI-VESC a los expertos, fue solicitada evaluación de los cuatro ítems que recibieron ajuste en la redacción por parte de los escolares. Las propuestas fueron aprobadas por los expertos y se obtuvieron sugerencias respecto a otros cuatro ítems que requirieron ajuste en su claridad y redacción (Ver Tabla II). 
Tabla II - Ajustes sugeridos por los expertos en cuatro enunciados de los ítems del SCSI. Colombia.

\begin{tabular}{cll}
\hline Ítem $\mathbf{n}^{\circ}$ & Ítems versión SCSI-VCON1 / SCSI-VESC & Propuesta de los expertos (SCSI-VEXP) \\
\hline & & \\
12 & Hago preguntas acerca de ello/ Hago preguntas sobre el tema & Hago preguntas sobre lo que me estresa. \\
16 & Doy golpes contra algo/Le pego a algo & Me alejo \\
22 & Disculparme/ Pido disculpas & Golpeo algo \\
\hline
\end{tabular}

* Solo hay una enunciado porque coincide en las dos versiones. Fuente: Briñez K, 2016

Con respecto al índice Kappa por cada indicador estimado se encontró, acuerdo casi perfecto en los indicadores de suficiencia: 0,81 , coherencia 0,80 y relevancia 0,92 ; acuerdo sustancial en los indicadores de claridad 0,77 y equivalencia semántica 0.76 , se obtuvo acuerdo del 90,2\%. Lo anterior identificó las cuatro sugerencias de los expertos a los ítems 1, 12,16 y 22 que fueron incluidas en la versión SCSI-VEXP.

Versión consenso final: En esta etapa, no se eliminó ningún ítem. Se analizaron las ocho modificaciones semánticas de los ítems propuestos entre los escolares y los expertos, los cuales se ajustaron al instrumento del SCSI y de este modo se obtuvo la versión SCSI-VCONF.

Prueba piloto: Participaron 19 escolares entre 11 y 13 años. De una institución educativa privada de lbagué de nivel socio económico medio, participaron seis de sexo femenino y 13 de sexo masculino pertenecientes a sexto grado de secundaria. Se confirmó la comprensión de los ítems y la facilidad para el diligenciamiento por escolares de la versión SCSI-VCONF. El tiempo para responder el instrumento fue entre 10 y 15 minutos.

Se analizó la confiabilidad mediante cálculo de alfa de Cronbach a la muestra piloto, con un resultado de 0,65 para la subescala de frecuencia y de 0.70 para la subescala de molestia. Para la escala total fue de 0,80 , los anteriores valores se consideraron como altos ${ }^{(23)}$.

\section{DISCUSIÓN}

El afrontamiento se considera importante en la calidad de vida de un individuo dada la integración entre él y su ambiente porque permite identificar la forma de responder a los cambios ${ }^{(6)}$. Por ejemplo, en escolares de 11 años, en quienes se usó el SCSI en versión portuguesa, se demostró que cuando usaban más estrategias de afrontamiento de regulación emocional negativas desarrollaban más problemas comportamentales y emocionales ${ }^{(10)}$.

Por lo anterior, es importante, que enfermería como disciplina de cuidado de la salud, genere conocimiento propio y cuente con instrumentos de medición como el SCSI, que faciliten la investigación del afrontamiento en diferentes lugares del mundo, de modo que los resultados sean comparables entre diferentes culturas para entender implicaciones en salud.

La necesidad de aplicar un instrumento de medición que mida estrategias de afrontamiento en edad escolar de habla hispana fue evidente, teniendo en cuenta que ninguno de los encontrados cumplía el requisito de ser creado para esta estas edades y menos ser de autoreporte.

El desarrollo de las versiones comprendió una serie de etapas que se fueron nutriendo a medida que el proceso avanzaba. Las traducciones oficiales VTES1 y VTES2 fueron realizadas a cargo de profesionales con sello institucional calificados originarios de Estados Unidos y Colombia que conocían los dos idiomas español e inglés; la sencillez del instrumento facilitó que no se usaran palabras técnicas por ser desarrollado para escolares, y se pudo identificar que la independencia en la realización de la traducciones facilitó dos versiones en español que se compararon posteriormente; lo anterior, sumado a la experticia profesional facilitó SCSI-VCOM con la elección de los ítems más comprensibles. Otros autores han recomendado que el retrotraductor debe conocer bien el idioma de origen, el instrumento debe ser claro en significado, y debe ser hábil en este proceso ${ }^{(12)}$, condiciones que se cumplieron en este estudio.

La validez se comprende como el grado en el que un instrumento refleja con precisión el concepto que se examina(20).

La validez de contenido es una etapa para la validación transcultural de instrumentos de medición. En este estudio fue realizada por los escolares y por los expertos. Esta etapa valora la pertinencia del contenido del ítem y 
la relevancia del ítem en el atributo a medir ${ }^{(26)}$. Las enfermeras investigadoras se han caracterizado por ser líderes en los métodos de este tipo de validez ${ }^{(24)}$.

Algunos estudios solo consideran que la participación de profesionales expertos es suficiente, pero en este estudio se analizó la relevancia de la construcción y adaptación que podía enriquecerse desde un juicio con personas de experiencia académica y de investigación ${ }^{(26)}$ concertado con un juicio desde la experiencia personal del concepto de los escolares. El proceso de validación transcultural debe ser metódico y sistemático ${ }^{(27)}$.

Una fortaleza de este estudio fue contar con escolares de niveles socioeconómicos alto y bajo en los grupos focales porque facilitó llegar a dos escenarios sociales sin que sea una limitación posterior para su desarrollo. Otros estudios han usado entrevistas semiestructuradas también en procesos de validación de instrumentos en niños ${ }^{(28)}$.

Las estrategias cualitativas en la validación de los ítems se logra para hacer el ítem sensible a la población destino ${ }^{(29)}$. En esta etapa los escolares sugirieron modificaciones en 4 ítems en algunas palabras facilitando su redacción. Este número pequeño de cambios confirma la sencillez y comprensibilidad del instrumento de medición.

Lo realizado en este estudio, es coherente con literatura Brasileña, que incluyó adolescentes y confirmó la importancia del análisis cualitativo mediante grupos focales como del cuantitativo con Kappa y alfa de Cronbach en los procesos de adaptación de instrumentos de medición ${ }^{(30)}$.

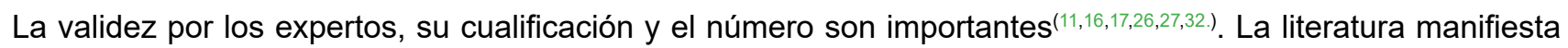
que hasta 10 expertos brindarían estimación confiable de la validez de contenido de un instrumento ${ }^{(22)}$. Este estudio contó con la participación de 6 expertos. Las sugerencias de ellos fueron en la redacción de cuatro ítems al hacer los ítems más personales y simples sin cambiar el sentido.

Otra fortaleza de esta etapa en el presente estudio es el alto porcentaje de acuerdo $(90,2 \%)$ entre los expertos en criterios de redacción, exactitud y claridad en el lenguaje de los ítems, lo que indicó que puede aplicarse el instrumento en escolares y en la investigación de estrategias de afrontamiento.

En la prueba piloto, el tiempo usado para el diligenciamiento del instrumento fue de 10 a 15 minutos y hubo facilidad en su aplicación. Se hizo análisis del alfa de Cronbach y los resultados evidenciaron consistencia, los valores se consideraron como altos ${ }^{(23)}$. Otro índice calculado, fue el Kappa, en el que criterios de equivalencia semántica 0,76 , claridad 0,77 obtuvieron evaluación de acuerdo sustancial; criterios de coherencia 0,80, suficiencia 0,81 y relevancia 0,92 obtuvieron evaluación de acuerdo casi perfecto. Esto mostró que la proporción de veces que los evaluadores estaban de acuerdo fue alta ${ }^{(26)}$.

Una limitación del estudio, fue el número de participantes en prueba piloto, sin embargo, en otros reportes la literatura indica que esta condición no invalida los resultados ${ }^{(12,16,27)}$. Lo anterior se justifica porque en este estudio los 19 escolares verificaron aspectos como practicidad, claridad y factibilidad del SCSI en el escenario escolar.

Este estudio aporta a la práctica de enfermería, en el cuidado de los escolares porque al ser autoreporte, permite conocer desde los escolares las estrategias de afrontamiento que usan y son más efectivas como respuestas cognitivas y comportamentales a diferentes estímulos. En el escenario clínico, las estrategias pueden correlacionarse con procesos agudos, crónicos, de recuperación de la salud, de enfermedad, de situación de discapacidad. En el escenario ambulatorio, pueden correlacionarse con actividades de cuidado propio y de prevención de enfermedades, con factores familiares de riesgo y protectores como violencia, pautas de crianza. En el escenario escolar pueden ser correlacionadas con fenómenos riesgosos para su salud (consumo de drogas, sexualidad, intimidación escolar) como con fenómenos de promoción de la salud. Los resultados pueden orientar intervenciones de cuidado de enfermería en proyectos de gestión hospitalarios, estrategias de salud pública, programas de educación a la familia, proyectos de salud escolar con el fin de que promuevan el bienestar y la adaptación de esta población, dado a que en la práctica, la enfermera es la profesional de salud que más tiene experiencia en las situaciones de cambio del ser humano(8). En el escenario docente, este estudio puede orientar a estudiantes y académicos en la importancia y necesidad de la investigación metodológica rigurosa para disponer de instrumentos de medición congruentes a las características propias de la edad escolar que aporten al conocimiento y la práctica.

\section{CONCLUSIÓN}

EI SCSI se validó transculturalmente para población colombiana. La versión obtenida fue el SCSI-VCONF y demostró ser comprensible para la población objeto, válido y confiable para la investigación en escolares para determinar la frecuencia del uso de las estrategias de afrontamiento y la efectividad de las mismas lo que constituye un aporte para futuras investigaciones en enfermería y otras disciplinas de la salud. 


\section{CONFLICTOS DE INTERESE}

Los autores declaran no tener conflictos de interese.

\section{CONTRIBUCIONES}

Karol Johanna Briñez Ariza contribuyó en revisión de literatura, redacción del artículo. Clara Virginia Caro Castillo y Marta Lenise do Prado contribuyeron en la redacción del artículo.

\section{REFERENCIAS}

1. Senger BA, Ward LD, Barbosa-Leiker C, Bindler RC. Stress and coping of parents caring for a child with mitochondrial disease. Appl Nurs Res [Internet]. 2016 [citado el 2016 Nov 3];29:195-201. Disponible en: http:// linkinghub.elsevier.com/retrieve/pii/S0897189715000701

2. Ered A, Gibson LE, Maxwell SD, Cooper S, Ellman LM. Coping as a mediator of stress and psychotic-like experiences. Eur Psychiatry [Internet]. 2017 [citado el 2017 Feb 14]; Disponible en: http://linkinghub.elsevier. com/retrieve/pii/S0924933817303425

3. Monat A, Lazarus RS, editores. Stress and coping: an anthology. $3^{\text {rd }}$ ed. New York: Columbia University Press; 1991.

4. Vandoninck S, d'Haenens L. Children's online coping strategies: Rethinking coping typologies in a risk-specific approach. J Adolesc [Internet]. 2015 [citado el 2017 Feb 14];45:225-36. Disponible en: http://linkinghub.elsevier. com/retrieve/pii/S0140197115002407

5. Almeida AC, Pereira MG. Psychometric properties of the portuguese version of the Coping Health Inventory for Parents (CHIP) of adolescents with chronic illness. J Pediatr Nurs [Internet]. 2016 [citado el 2017 Feb 14];31(5):528-36. Disponible en: http://linkinghub.elsevier.com/retrieve/pii/S0882596316300409

6. Roy C, Bakan G, Li Z, Nguyen TH. Coping measurement: creating short form of Coping and Adaptation Processing Scale using item response theory and patients dealing with chronic and acute health conditions. Appl Nurs Res [Internet]. 2016 [citado el 2017 Fev];32:73-9. Disponible en: http://linkinghub.elsevier.com/retrieve/pii/ S0897189716300234

7. Roy C. The Roy adaptation model. $3^{\text {rd }}$ ed. Upper Saddle River: Pearson Prentice Hall; 2009.

8. Roy C, Roy Adaptation Association. Generating middle range theory: from evidence to practice. New York: Springer Pub; 2014.

9. Ryan-Wenger NM. Development and psychometric properties of the schoolagers coping strategies inventory. Nurs Res [Internet]. 1990 [citado el 2015 Mar 28];39(6):344-9. Disponible en: http://content.wkhealth.com/linkback/ openurl?sid=WKPTLP:landingpage\&an=00006199-199011000-00005

10. Conceição A, Carvalho M. Emotional and behavioral problems in preadolescents: relationships with temperament, coping and emotional regulation strategies, and facial expressions identification. Psychologica [Internet]. 2013 [citado el 2016 Nov 3];56:83-100. Disponible en: https://digitalis.uc.pt/handle/10316.2/29957

11. De Luna-Rodríguez ME, Aranda-Gallardo M, Canca-Sánchez JC, Vazquez-Blanco MJ, Moya-Suárez AB, MoralesAsencio JM. Cross-cultural adaptation of the STRATIFY tool in detecting and predicting risk of falling. Enferm Clínica Engl Ed [Internet]. 2017 [citado el 14 de febrero de 2017];27(2):101-5. Disponible en: http://linkinghub. elsevier.com/retrieve/pii/S2445147916300108

12. Ferreira L, Nogueira A, Betanho M, Gomes M. Guia da AAOS/IWH: sugestões para adaptação transcultural de escalas. Aval Psicológica [Internet]. 2014 [citado el 2016 Oct 2016];13(3):457-61. Disponible en: http://pepsic. bvsalud.org/pdf/avp/v13n3/v13n3a18.pdf

13. Lima L, Serra LM, Prista M. Estudo das qualidades psicométricas do SCSI (Schoolagers' Coping Strategies Inventory) numa população portuguesa. Anál Psicol. 2002;20(4):555-70.

14. Ryan N. Directions for use of the Schoolagers' Coping Strategies Inventory (SCSI). 2014. 
15. Naar-King S, Ellis DA, Frey MA, editores. Assessing children's well-being: a handbook of measures. Mahwah: Lawrence Erlbaum Associates; 2004.

16. Lauffer A, Solé L, Bernstein S, Lopes MH, Francisconi CF. Cómo minimizar errores al realizar la adaptación transcultural y la validación de los cuestionarios sobre calidad de vida: aspectos prácticos. Rev Gastroenterol México [Internet]. 2013 [citado el 2016 Sep 16];78(3):159-76. Disponible en: http://linkinghub.elsevier.com/ retrieve/pii/S0375090613000529

17. Cukljek S, Juresa V, Babic J. The cross-cultural (transcultural) adaptation and validation of the nursing image questionnaire. Nurse Educ Today [Internet]. 2017 [citado el 2017 Feb 14];48:67-71. Disponible en: http:// linkinghub.elsevier.com/retrieve/pii/S0260691716302003

18. Ministerio de Salud (CO). Resolución № 008430 DE 1993. Por la cual se establecen las normas científicas, técnicas y administrativas para la investigación en salud. [Internet]. 1993 [citado el 2017 Feb 14]. Disponible en: http://www.dib.unal.edu.co/promocion/etica_res_8430_1993.pdf

19. Escobar J, Bonilla F. Grupos focales: una guía conceptual y metodológica. Cuad Hispanoam Psicol. 2009;9(1):5167.

20. Burns N, Grove SK, Gray J. Investigación en enfermería: desarrollo de la práctica enfermera basada en la evidencia. Amsterdam: Elsevier; 2012.

21. Hamui A, Varela M. La técnica de grupos focales. Investig En Educ Médica [Internet]. 2013 [citado el 2016 Oct 2016];2(1):55-60. Disponible en: http://riem.facmed.unam.mx/sites/all/archivos/V2Num01/09_MI_HAMUI.PDF

22. Escobar J, Cuervo A. Validez de contenido y juicio de expertos: una aproximación a su utilización. Av En Medición [Internet]. 2008 [citado el 2016 Oct 2016];6(1):27-36. Disponible en: http://www.humanas.unal.edu.co/psicometria/ files/7113/8574/5708/Articulo3_Juicio_de_expertos_27-36.pdf

23. Bowling A. Measuring health: a review of quality of life measurement scales [Internet]. New York: Open University Press; 2005 [citado el 2016 Oct 2016]. Disponible en: http://public.eblib.com/choice/publicfullrecord.aspx?p=287844

24. Polit DF. Assessing measurement in health: Beyond reliability and validity. Int J Nurs Stud [Internet]. 2015 [citado el 2017 Feb 7];52(11):1746-53. Disponible en: http://linkinghub.elsevier.com/retrieve/pii/S0020748915002242

25. LoBiondo-Wood G, Haber J. Nursing research. Methods and critical appraisal for evidence-based practice. $8^{\text {th }}$ ed. Elsevier; 2014.

26. Parrado lozano YM, Sáenz Montoya X, Soto Lesmes VI, Guáqueta Parada SR, Amaya Rey P, Caro Castillo $\mathrm{CV}$, et al. Validez de dos instrumentos para medir la relación interpersonal de la enfermera con el paciente y su familia en la unidad de cuidado intensivo. Investig En Enferm Imagen Desarro [Internet]. 2016 [citado el 2017 Feb 7];18(1):115. Disponible en: http://revistas.javeriana.edu.co/index.php/imagenydesarrollo/article/view/15069

27. Calvo J, Sanchez-Pedraza R, Peña-Solano D. Traducción y adaptación de la escala SQLS para medir calidad de vida en pacientes con esquizofrenia en Colombia. Rev Fac Med [Internet]. 2012 [citado el 2017 Feb 7];60(2):95102. Disponible en: http://www.revistas.unal.edu.co/index.php/revfacmed/article/view/36012/47220

28. González-Arratia-López-Fuentes NI, Valdez Medina JL. Validez de la Escala de Salud Mental Positiva en Niños Mexicanos. Acta Investig Psicológica [Internet]. 2016 [citado el 2017 Feb 14];6(1):2368-83. Disponible en: http:// linkinghub.elsevier.com/retrieve/pii/S2007471916300564

29. Hartley JEK, Levin K, Currie C. A new version of the HBSC Family Affluence Scale - FAS III: Scottish Qualitative Findings from the International FAS Development Study. Child Indic Res [Internet]. 2016 [citado el 2017 Feb 14];9(1):233-45. Disponible en: http://link.springer.com/10.1007/s12187-015-9325-3

30. Paiva PCP, Paiva HN, Oliveira PM Filho, Lamounier JA, Ferreira EF, Ferreira RC, et al. Development and Validation of a Social Capital Questionnaire for Adolescent Students (SCQ-AS). PLoS ONE [Internet]. 2014 [citado el 2017 Feb 14];9(8):e103785. Disponible en: http://dx.plos.org/10.1371/journal.pone.0103785

31. Caro-Bautista J, Morilla-Herrera JC, Villa-Estrada F, Cuevas-Fernández-Gallego M, Lupiáñez-Pérez I, MoralesAsencio JM. Adaptación cultural al español y validación psicométrica del Summary of Diabetes Self-Care Activities measure (SDSCA) en personas con diabetes mellitus tipo 2. Atención Primaria (Barcelona) [Internet]. 2016 [citado el 2016 Nov 7];48(7):458-67. Disponible en: http://linkinghub.elsevier.com/retrieve/pii/S0212656715003388 
32. Silveira ALS, Grassi-Oliveira R. Semantic validation of the ISPCAN Child Abuse Screening Tools (ICAST) in Brazilian Portuguese. Trends Psychiatry Psychother [Internet]. 2016 [citado el 2016 Nov 26];38(2):105-10. Disponible en: http://www.scielo.br/scielo.php?script=sci_arttext\&pid=S2237-60892016000200105\&Ing=en\&n $\mathrm{rm}=$ iso\&tlng=en

Dirección para correspondencia:

Karol Johanna Briñez Ariza

Pontificia Universidad Javeriana

Carrera 7, No 40-62

Bogotá D. C. - Colombia

E-mail: kjbrineza@unal.edu.com 